\title{
AVALIAÇÃO DE POLUIÇÃO DO AR POR CHUMBO PARTICULADO: UMA ABORDAGEM GEOQUÍMICA
}

\author{
Argeu Vanz", Nicolai Mirlean* e Paulo Baisch \\ Departamento de Geociências, Fundação Universidade do Rio Grande, Av. Itália km 08, Campus Carreiros, 96201-900 Rio Grande \\ - RS
}

Recebido em 21/11/01; aceito em 8/7/02

\begin{abstract}
EVALUATION OF THE AIR POLLUTION BY PARTICULATE LEAD: GEOCHEMICAL APPROACH. High lead concentrations up to $5478 \mathrm{mg} \mathrm{kg}^{-1}$ were detected in dry atmospheric precipitations in Rio Grande city and adjacent area. Lead anomalies cover up to $30 \%$ of the study area. Lead concentration measurement in dry atmospheric precipitation and suspended particulate matter provides a possibility to detect concentrations above lead threshold in the air. The most impacted areas are fishermen's villages. The most possible source of lead in the air is uncontrolled sub industrial activity of local population
\end{abstract}

Keywords: lead; precipitation; air pollution.

\section{INTRODUÇÃO}

O chumbo é reconhecido pela Organização Mundial da Saúde como um dos elementos químicos mais perigosos para a saúde humana ${ }^{1}$. Após a proibição parcial ou total da adição do chumbo tetraetil na gasolina de alguns países, a concentração do chumbo particulado no ar das zonas urbanas diminuiu, mas não determinou o desaparecimento do problema da poluição por esse metal $^{2}$. Outras fontes são responsáveis pela poluição atmosférica por chumbo particulado nas zonas industriais e urbanas, colocando em risco a saúde das populações. Em muitos países, o chumbo é o único metal cuja presença no ar é controlada por legislação ${ }^{3}$.

O método mais conhecido de controle de poluição atmosférica por chumbo é o da filtração por bombeamento de um volume determinado de ar para posterior análise dos filtros ${ }^{4,5}$. Este método é utilizado por órgãos oficiais de vários países para o controle da poluição do ar em zonas urbanas e industriais, mas em razão de dificuldades operacionais, freqüentemente registra-se a poluição em um número reduzido de pontos de monitoramento. Uma alternativa ao acima descrito é o emprego de um método de estudo das Precipitações Sólidas Atmosféricas (PSA), através de uma abordagem geoquímica. No método geoquímico pode-se utilizar a amostragem da superfície da neve ou do solo, assim como de coletores passivos artificiais ${ }^{6-8}$. Os métodos geoquímicos de avaliação não substituem integralmente os métodos tradicionais, mas apresentam algumas vantagens, como a possibilidade da instalação de um grande número de pontos de coletas nas áreas investigadas. Esse aspecto permite caracterizar de forma mais detalhada a distribuição espacial da poluição e facilita a identificação das anomalias e suas fontes ${ }^{6,8}$.

As estatísticas dos resultados de estudos geoquímicos em zonas urbanas e de dados do quadro clínico das populações permitiram estabelecer critérios geoquímicos de avaliação da intensidade de poluição atmosférica ${ }^{7}$. Entre esses parâmetros, podem ser citados o limite de precipitação do material particulado (200 kg km${ }^{-2}$ dia) e da concentração de chumbo na PSA (1500 mg kg-1), os quais se ultrapassados considera-se que provoquem efeitos negativos à saúde humana $^{7,9-11}$.

\footnotetext{
*e-mail: NMirlean@geo1.log.furg.br

\# Endereço atual: Centro Tecnológico, Engenharia Sanitária e Ambiental, Universidade Federal de Santa Catarina, 88010-970 Florianópolis - SC
}

No Brasil não existem parâmetros que limitem a concentração do chumbo no ar, assim como não existem normas específicas para o seu controle na atmosfera.

Este trabalho tem por objetivo avaliar a poluição por chumbo particulado na atmosfera, através de uma abordagem geoquímica na região urbana e industrial da cidade do Rio Grande. Esta região apresenta alta densidade de construções civis e possui um longo histórico de impacto atmosférico por poeira e metais particulados, causado por atividades portuárias e industriais, especialmente do setor de fertilizantes, refino de petróleo, alimentos, entre outras ${ }^{8,12,13}$ (Figura 1).

O presente trabalho destaca a existência de um problema de poluição atmosférica por chumbo particulado no município de Rio Grande que, devido à sua importância, deverá ser motivo de novas investigações.

\section{PARTE EXPERIMENTAL}

A amostragem do material particulado atmosférico foi efetuada na região emersa das áreas de assentamentos industriais, áreas urbanas e da região estuarina da Lagoa dos Patos. Os coletores foram construídos e testados no Laboratório de Oceanografia Geológica, para a amostragem de partículas de tamanho entre 500 e $0,45 \mu \mathrm{m}$.

$\mathrm{Na}$ confecção dos coletores foram utilizados materiais inertes como funis de polipropileno, canos e peças em PVC (Figura 2). A membrana de acetato de celulose $\left(0,45 \mu \mathrm{m}\right.$ Millipore $\left.{ }^{\circledR}\right)$ foi fixada entre duas arruelas de polipropileno, sendo inserida em um suporte e conectada um cano, que faz a ligação com o funil coletor. Esse conjunto era integralmente removido durante a tomada das amostras.

A parte superior do coletor consiste em um funil de polipropileno (30 cm de diâmetro), prolongado por um cilindro de $25 \mathrm{~cm}$ de altura, para evitar a remoção pelo vento de partículas atmosféricas já coletadas. A borda superior do cilindro foi serrilhada com a função de impedir a contaminação das amostras pelos pássaros.

Para impedir que insetos e partículas de grande tamanho chegassem ao filtro, foi colocada uma rede de nylon de $500 \mu \mathrm{m}$, presa em anéis de teflon do mesmo diâmetro interno da haste do funil. Um canudo plástico foi fixado sobre a rede, para evitar a formação de bolhas de ar, que poderiam causar a redução da velocidade de filtração e, assim, favorecer o desenvolvimento de algas.

Todos os materiais utilizados no coletor foram analisados quanto aos teores de chumbo, sendo considerados livres desse metal. 

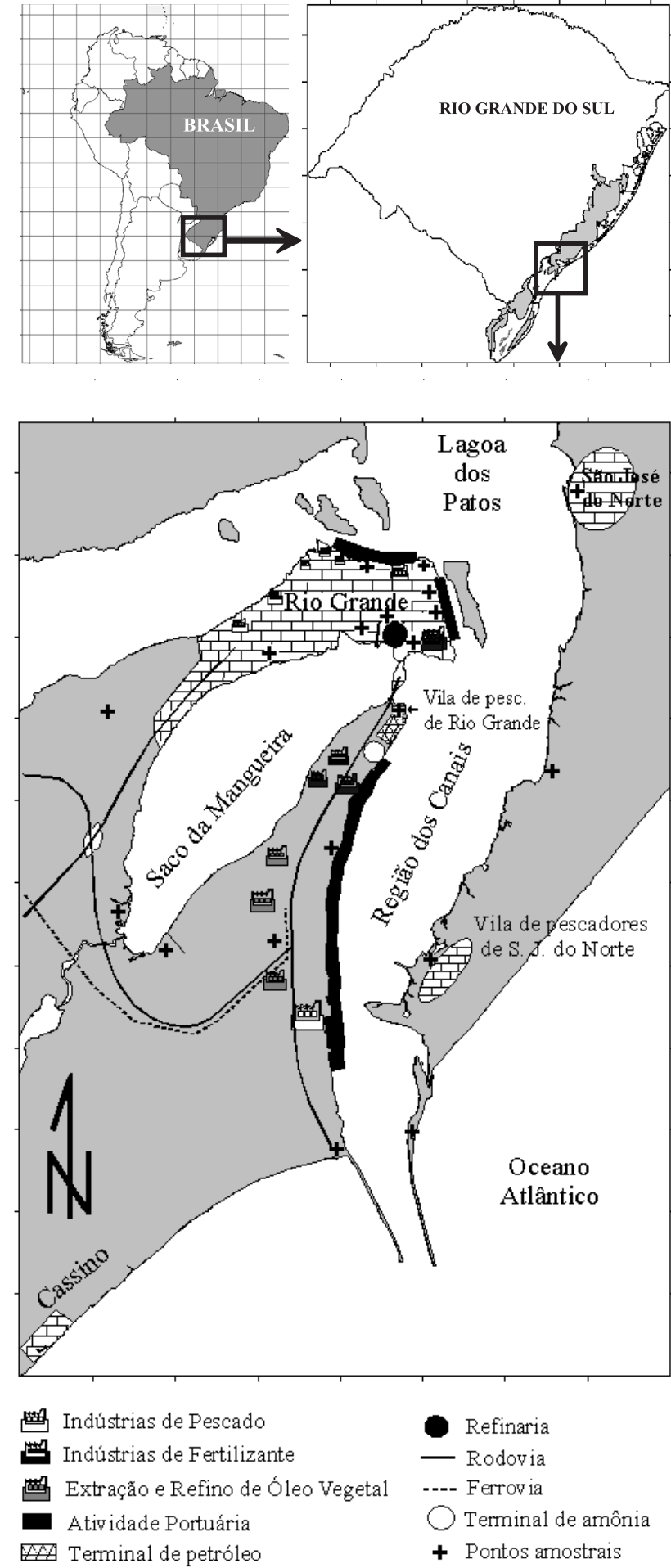

Figura 1. Localização da área de estudo, incluindo os pontos amostrais e os principais assentamentos industriais, urbanos e portuários

As unidades de coleta foram previamente lavadas com $\mathrm{HNO}_{3}$ $2 \%$ e água destilada. Antes das coletas, as membranas de acetato de celulose foram lavadas com $\mathrm{HNO}_{3} 10 \%$ e água Milli-Q. Posteriormente foram secas, pesadas $( \pm 0.1 \mathrm{mg})$ e ajustadas nas unidades de coleta ainda no laboratório. As unidades de coleta foram transportadas até os pontos de coleta em sacos plásticos. A parte interna do funil e cilindro dos coletores foi lavada diversas vezes com água destilada antes de ser fixada à unidade de coleta.

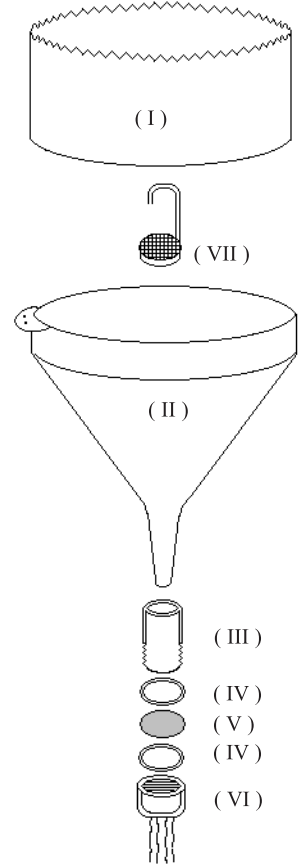

Figura 2. Partes principais do coletor de PSA : I - cilindro; II - funil de polipropileno (diâmetro $30 \mathrm{~cm}$ ); III - cano; IV - arruelas de polipropileno; $V$ - membrana de acetato de celulose $(0,45 \mu \mathrm{m})$; VI - suporte de PVC; VII rede de nylon de 500 um com canudo plástico

Foram instalados 19 coletores (Figura 1) em construções civis a uma altura aproximada de $5 \mathrm{~m}$ do solo, correspondendo a $1,5 \mathrm{~m}$ acima dos telhados para evitar contaminações locais. Efetuou-se uma coleta em cada estação do ano, de maio de 1998 a fevereiro de 1999 , com uma exposição máxima dos coletores de 30 dias por amostragem.

Durante a tomada das amostras, a unidade de coleta foi acondicionada em sacos plásticos e a superfície interna do coletor foi lavada com água destilada para remoção do material particulado aderido. A água recolhida foi colocada em frascos plásticos previamente limpos. No laboratório esta água foi passada pelo respectivo filtro.

As membranas com o material coletado foram colocadas em placas de Petri para secagem à temperatura ambiente, e em seguida foram pesadas para determinar a quantidade de material.

As membranas juntamente com material foram digeridos em $3 \mathrm{ml}$ de água régia $\left(\mathrm{HCl}+\mathrm{HNO}_{3}-3: 1\right)$ em bomba de teflon-inox, por $3 \mathrm{~h}$ a $100{ }^{\circ} \mathrm{C}$. Ao extrato da digestão na bomba foi adicionado $20 \mathrm{ml}$ de água Milli-Q, sendo a seguir filtrado (Millipore ${ }^{\circledR} 0,45 \mu \mathrm{m}$ ), e a solução resultante foi levada à evaporação em chapa quente em béquer de teflon. Os filtros com o resto do material não digerido na bomba foram incinerados em mufla em $550{ }^{\circ} \mathrm{C}$. As cinzas foram digeridas com $2 \mathrm{ml}$ de água régia e $2 \mathrm{ml}$ de $\mathrm{HF} 40 \%$, durante $3 \mathrm{~h}$ à $100{ }^{\circ} \mathrm{C}$. O extrato resultante dessa etapa foi adicionado ao extrato da primeira etapa, sendo evaporado até quase a secura em béquer de teflon. O material resultante foi diluído com $25 \mathrm{ml} \mathrm{HNO}_{3} 2 \%$, e aferido a $50 \mathrm{ml}$ em balão volumétrico com água Milli-Q.

O chumbo foi dosado por espectrofotometria de absorção atômica, utilizando-se um aparelho GBC 932AA com Forno de Grafite (GF3000). A validade do método analítico e o desempenho do equipamento para dosagem de chumbo foram testados pelo exame de amostras padrões internacionais de sedimentos ${ }^{14}$. Todas medidas de chumbo foram feitas com controle de reprodutividade entre as réplicas com desvio padrão menor que $6 \%$.

Para o valor de "background" atmosférico do chumbo foi utilizado o teor do elemento na camada superficial do solo local, segun- 
do procedimentos metodológicos previamente propostos ${ }^{6,7}$. Para tanto, foram coletadas em zonas afastadas e livres de contaminação, 3 amostras superficiais de solos (Planossolos) de textura arenosa, os quais são os mais representativos da região ${ }^{15}$. $\mathrm{O}$ valor da média aritmética da concentração de chumbo das três amostras foi utilizado como "background" atmosférico nesse trabalho.

Para examinar a distribuição granulométrica do chumbo, procedeu-se à análise da fração inferior a $300 \mu \mathrm{m}$ (peneirado), que correspondia ao tamanho das partículas que contém cerca de $90 \%$ de chumbo encontrado no PSA. Para o fracionamento granulométrico do material particulado atmosférico total foram empregadas redes de nylon de 10, 20, 50, 150, $300 \mu \mathrm{m}$. Esse procedimento foi realizado em amostras tomadas por meio de coletores conectados com balões de 101 para coleta ("bulk precipitation") nos mesmos pontos de coleta. A separação das frações foi realizada a úmido utilizando-se a água da chuva coletada com material particulado. O material fracionado foi digerido e dosado segundo os procedimentos acima descritos.

\section{RESULTADOS E DISCUSSÃO}

A intensidade das precipitações sólidas atmosféricas na região da cidade do Rio Grande durante o período de estudo variou de 31,4 até $1901,5 \mathrm{~kg} \mathrm{~km}^{-2} \mathrm{dia}^{-1}$, perfazendo uma média geométrica de 177,1 $\mathrm{kg} \mathrm{km}^{-2} \mathrm{dia}^{-1}$, DPR-166\%. Em 20\% dos casos o valor de precipitação do material particulado superou o limite permitido para precipitação sólida (200 kg km${ }^{-2}$ dia) estabelecido nos países do leste europeu ${ }^{11}$.

O "background" do chumbo estabelecido pela fração granulométrica dos solos inferior a $300 \mu \mathrm{m}$ é menor do que o valor do "background" mundial e está mais próximo dos valores dos arenitos, refletindo a composição mineralógica dos solos regionais, essencialmente de textura arenosa (Tabela 1).

Tabela 1. Valores de "background" $\left(\mathrm{mg} \mathrm{kg}^{-1}\right)$ do chumbo no presente trabalho comparados aos estabelecidos para solos, arenitos e sedimentos mundiais

\begin{tabular}{ccccc}
\hline Localização & $\begin{array}{c}\text { Rio Grande } \\
\text { solos }\end{array}$ & $\begin{array}{c}\text { Mundial } \\
\text { solos }^{17}\end{array}$ & $\begin{array}{c}\text { Mundial } \\
\text { arenitos }^{18}\end{array}$ & $\begin{array}{c}\text { Mundial } \\
\text { sedimentos }^{19}\end{array}$ \\
\hline $\mathrm{Pb}$ & 13,6 & 29 & 10 & 19 \\
\hline
\end{tabular}

Quando se compara as concentrações de chumbo no PSA com o "background" dos solos regionais verifica-se que todas as amostras do material particulado apresentam valores mais elevados. A concentração do chumbo no PSA variou durante o período de estudo entre 177,4 e $5478,7 \mathrm{mg} \mathrm{kg}^{-1}$, com uma média geométrica de $855,2 \mathrm{mg}$ $\mathrm{kg}^{-1}, \mathrm{DPR}-83 \%$. O número de amostras com teores de chumbo acima $1500 \mathrm{mg} \mathrm{kg}^{-1}$ foi de $23 \%$. Verifica-se que valores iguais ou superiores a esse limite foram encontrados nas quatro estações do ano, recobrindo áreas de 5 a $30 \%$ da zona urbana municipal (Figura 3).

A distribuição do chumbo nas frações granulométricas das PSA corresponde à condição natural em relação aos metais pesados ${ }^{7}$, ou seja, existe um aumento de concentração com diminuição do tamanho das partículas (Figura 4). Observa-se que mais de 70\% do chumbo está associado às partículas de tamanho menores de $150 \mu \mathrm{m}$, e cerca de $35 \%$ às partículas de tamanho inferior a $10 \mu \mathrm{m}$.

As concentrações de Chumbo nas PSA acima de $1500 \mathrm{mg} \mathrm{kg}^{-1}$ delimitam as regiões onde podem ser registrados riscos à saúde da população, pois estão acima dos limites sanitários ${ }^{11}$. Em alguns pontos das áreas urbanizadas esse valor é superado algumas vezes, com agravante de se manterem ao longo do tempo, como pode ser observado na Figura 3. Outras partes da cidade apresentam teores menores, entre 500 e $1500 \mathrm{mg} \mathrm{kg}^{-1}$ de chumbo, contudo também se enqua-

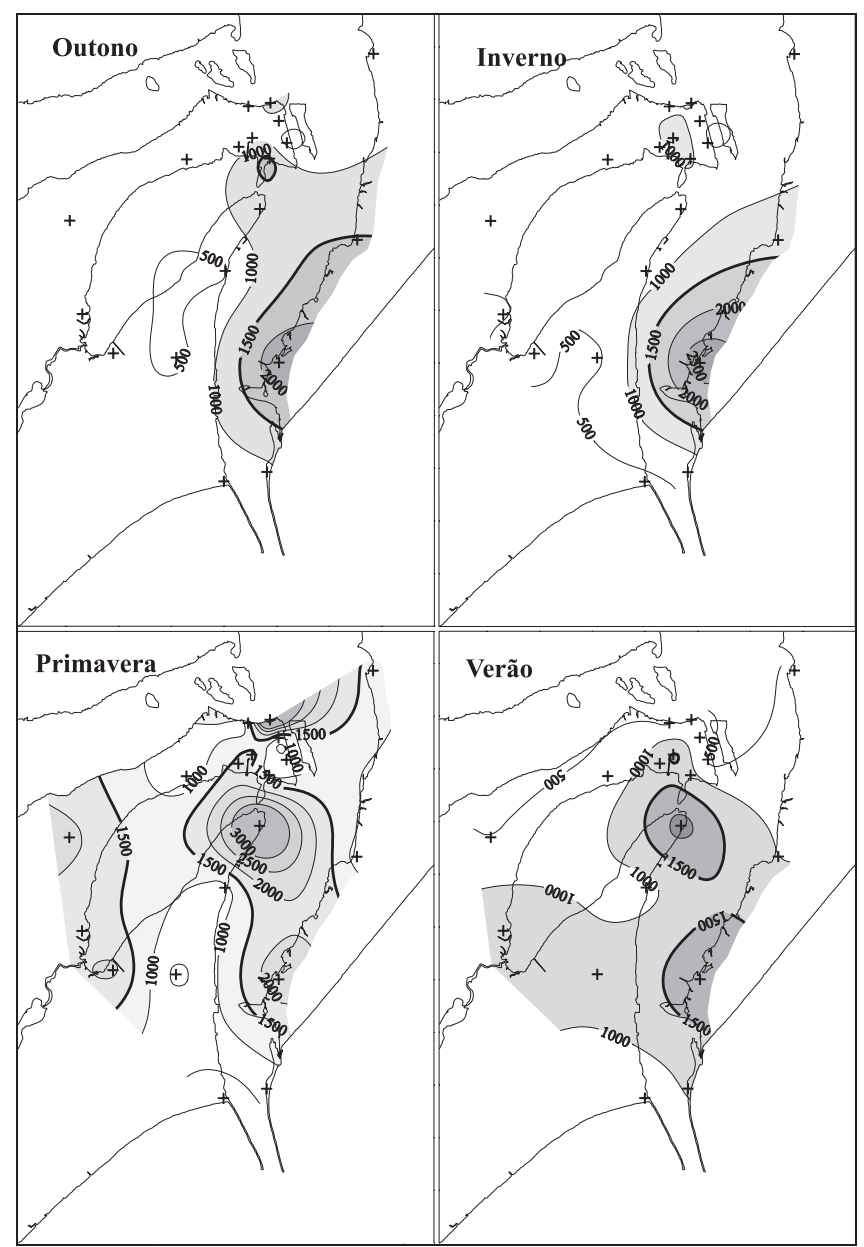

Figura 3. Distribuição espacial das concentrações de chumbo $\left(\mathrm{mg} \mathrm{kg}^{-1}\right)$ nas estações do ano

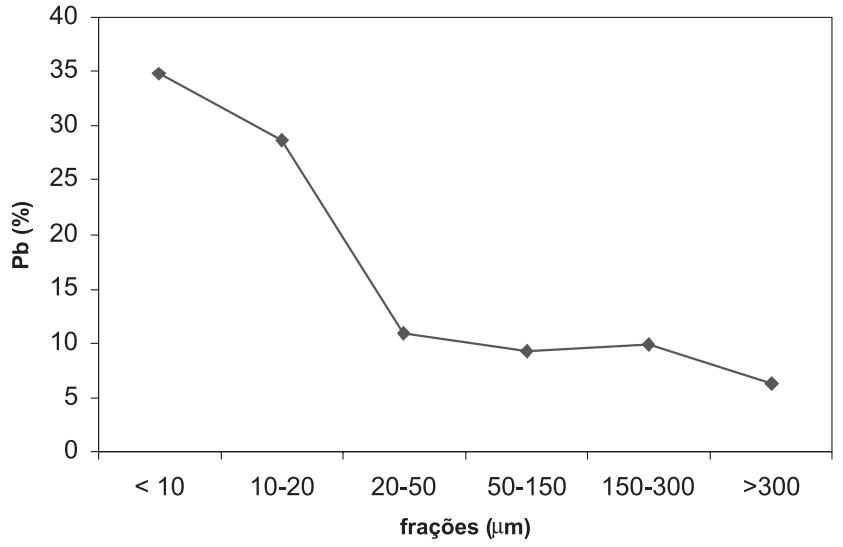

Figura 4. Distribuição percentual dos teores de chumbo em diferentes frações granulométricas das PSA

dram dentro de um processo de poluição atmosférica. Esses valores não atingem o limite máximo permitido, mas se inserem dentro de um nível considerado crítico. Em razão de sua associação com o material superficial do solo $^{16}$, o chumbo particulado depositado na superfície do solo pode ser a causa de doenças específicas, graças ao hábito da geofagia comum entre as crianças? 
A presença de altas concentrações em zonas afastadas dos principais assentamentos urbanos, portuários e industriais (Figuras $1 \mathrm{e}$ 3) indica que o chumbo apresenta, na região estuarina da Lagoa dos Patos, uma fonte com característica difusa e não tradicional ${ }^{17,18}$.

Não foi possível identificar as fontes de chumbo atmosférico nesse trabalho, contudo podem ser levantadas algumas hipóteses. A hipótese considerada mais consistente está ligada à atividade das populações relacionada à pesca. A fusão de chumbo para confecção de utensílios de pesca pode levar ao aparecimento de altas concentrações do chumbo nas PSA. Durante essa preparação, a escoria de óxidos de chumbo não utilizada, é descartada sobre a superfície do solo, resultando em uma fonte secundária desse metal na forma de particulado para o ar. Esse fato pode explicar a anomalia permanente com alta concentração de chumbo na Vila dos Pescadores.

Outra possibilidade de uma fonte adicional para a atmosfera poderia estar relacionada à tinta, contendo óxido de chumbo, das construções mais antigas. As partículas seriam produzidas pela abrasão eólica nas construções como resultado do intenso regime de ventos da região. A Vila dos Pescadores estaria mais susceptível a esse fenômeno devido à presença de prédios de pequeno tamanho, além de estar construída ao largo de grandes campos de areias.

Nos estudos de poluição do ar, os parâmetros geoquímicos podem servir como uma maneira de se obter informações suplementares aos resultados dos métodos previstos na legislação ambiental. Em termos ambientais, a concentração de chumbo na poeira mais fina é a mais problemática à saúde pública, pois pode atingir as partes internas dos órgãos respiratórios. Por essa razão, a granulometria das partículas é muito importante na determinação da intensidade da poluição do ar por chumbo. Para esse tipo de monitoramento, a EPA adota, em legislação, o limite máximo de $1,5 \mu \mathrm{g} \mathrm{m}^{-3}$ de chumbo nas partículas de tamanho inferior a $10 \mu \mathrm{m}\left(\mathrm{PM}_{10}\right)$, enquanto os países do leste europeu ${ }^{11}$ adotam o valor de $0,7 \mu \mathrm{g} \mathrm{m}^{-3}$.

Como a composição e os teores das partículas no ar são resultantes de um mesmo processo ambiental em uma determinada área, torna-se possível o emprego dos resultados geoquímicos para estimar as concentrações de chumbo referentes aos dados do material particulado efetuados pela FEPAM $^{4}$.

Os resultados do monitoramento do material particulado no ar inferior a $100 \mu \mathrm{m}\left(\mathrm{PM}_{100}\right)$ feitos pela FEPAM 4 em Rio Grande mostram uma significativa variação desse parâmetro durante os últimos 9 anos, oscilando entre 4,0 a 1165,0 $\mu \mathrm{g} \mathrm{m}^{-3}$ (média $-73.7 \mu \mathrm{g} \mathrm{m}^{-3}$, DPR - 160,7\%).

Se admitirmos que a composição global do material particulado coletado nos filtros da FEPAM ${ }^{4}$ é a mesma do material acumulado nos filtros dos coletores do presente estudo, o cálculo da concentração de chumbo no ar pode ser feito pela seguinte formula:

$\mathrm{C}_{\mathrm{Pb}(\text { ar })}=\mathrm{K}_{1} * \mathrm{~A} * \mathrm{~K}_{2} * \mathrm{C}_{\mathrm{Pb}(\mathrm{PSA})}$, onde

$\mathrm{C}_{\mathrm{Pb}(\mathrm{ar})}$ - concentração do chumbo particulado no ar em partículas de tamanho inferior $10 \mu \mathrm{m}$, em $\mu \mathrm{g} \mathrm{m}^{-3}$;

$\mathrm{K}_{1}$ - coeficiente proporcional da presença das partículas inferiores a $10 \mu \mathrm{m}$ nos filtros de coletas da FEPAM ${ }^{4}$, obtido com base nos resultados de estudo de distribuição das frações granulométricas das PSA, nesse caso $=0,23$;

A - concentração $\left(\mu \mathrm{g} \mathrm{m}^{-3}\right)$ do material particulado (inferior a $100 \mu \mathrm{m}$ ) no ar - medidas FEPAM ${ }^{4}$;

$\mathrm{K}_{2}$ - coeficiente de proporcionalidade da concentração de chumbo nas partículas inferior a $10 \mu \mathrm{m}$ e concentração total de chumbo nas amostras das PSA (ver Figura 4), neste caso = 2,05;

$\mathrm{C}_{\mathrm{Pb}(\mathrm{PSA})}$ - concentração de chumbo nas PSA, em $\mu \mathrm{g} \mathrm{mg}^{-1}$.

Os resultados do cálculo das concentrações possíveis de chum- bo particulado no ar do Rio Grande encontram-se representados graficamente na Figura 5. Esse gráfico apresenta o campo de ocorrência das concentrações de chumbo para as partículas de tamanho inferior a $10 \mu \mathrm{m}$, pois essa é a fração mundialmente empregada para esse tipo de monitoramento. Os campos das concentrações do chumbo acima dos limites admitidos estão delimitados pelas linhas indicativas dos teores máximos de chumbo no ar $\left(\mathrm{Pb}^{\mathrm{PM}} \mathrm{M}_{10}\right)$ recomendados pela $\mathrm{EPA}^{5}$ e pelos países do leste europeu ${ }^{11}$.

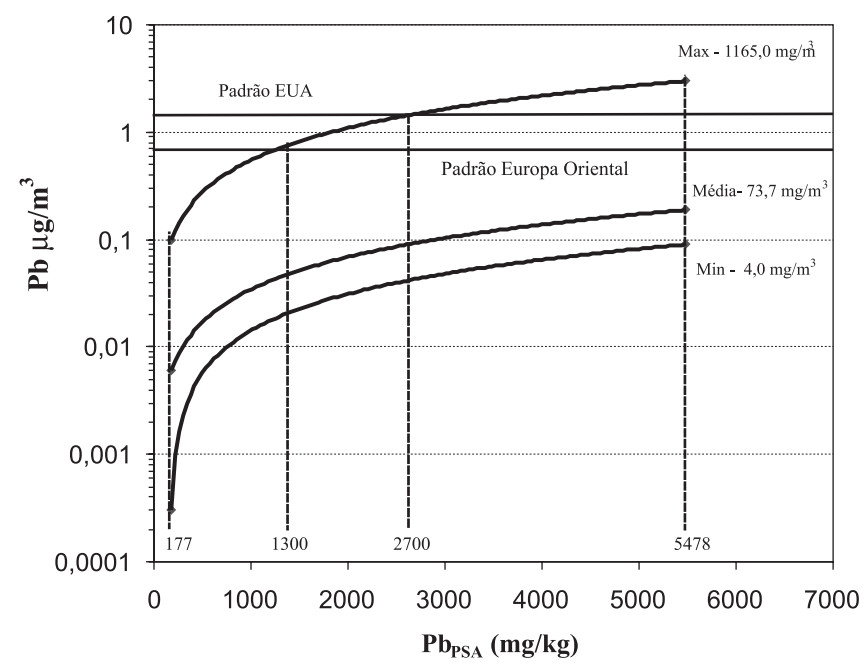

Figura 5. Estimativa dos teores de chumbo no $P M_{10}$ através de sua concentração nas precipitações sólidas atmosféricas. As curvas representam as concentrações de $\mathrm{PbPM}_{10}$ para os valores máximo, médio e mínimo do $P M_{100} . P P_{10}$ padrão(USA) - 1,5 $\mu \mathrm{g} \mathrm{m}^{-3} ; \mathrm{PbPM}_{10}$ padrão (Europa Oriental) $-0,7 \mu \mathrm{g} \mathrm{m} \mathrm{m}^{-3}$

A relação estabelecida nesse trabalho entre o teor de chumbo do particulado total e do $\mathrm{PM}_{10}$ permite estabelecer que, sob condições de máximos valores de material particulado, o valor legal de qualidade do ar $\left(1,5 \mu \mathrm{g} \mathrm{m}^{-3}\right)$ nos USA é atingido nas áreas onde forem encontradas concentrações acima de $2700 \mathrm{mg} \mathrm{kg}^{-1}$ de chumbo na PSA. Nessas mesmas condições, pela legislação dos países da Europa Oriental, o valor de qualidade do ar é atingido $\left(0,7 \mu \mathrm{g} \mathrm{m}^{-3}\right)$ se os teores de chumbo no PSA ultrapassarem $1300 \mathrm{mg} \mathrm{kg}^{-1}$.

Há fortes indícios de que, sob certas condições, os limites legais de controle são ultrapassados e deste modo pode representar perigo de contaminação às populações. Contudo há necessidade da comprovação desse quadro ambiental, através da análise de chumbo diretamente no $\mathrm{PM}_{10}$.

\section{CONCLUSÃO}

Os dados obtidos por esse estudo mostram que existe um processo de contaminação atmosférica por chumbo na cidade do Rio Grande e na região estuarina da Lagoa dos Patos. Em algumas regiões da cidade os teores de chumbo das precipitações sólidas atmosféricas superam, em algumas vezes, os limites legais estabelecidos para países da Europa Oriental.

As características apresentadas pela poluição do chumbo nas PSA não permitem classificá-la como sendo provocada por fontes industriais. Provavelmente sua origem está relacionada às atividades ligadas ao preparo de materiais para a arte da pesca ou outras atividades ainda não identificadas desse grupo populacional. Esse quadro ambiental poderá ser encontrado em outras cidades brasileiras, onde a estrutura das atividades sócio-econômicas for semelhante. 
A análise dos dados de chumbo nas PSA em relação aos teores do material particulado do ar permitiu estimar a possibilidade de encontrar valores no ar acima dos limites legais estabelecidos nos EUA e nos países da Europa Oriental. Na cidade do Rio Grande e região estuarina há fortes indicativos que os limites legais de qualidade de ar em relação ao chumbo sejam ultrapassados sob condições de altos teores de material particulado atmosférico associado a altos teores desse metal.

As populações das áreas urbanizadas das vilas dos pescadores artesanais são as que apresentam maior risco em relação a uma possível contaminação por chumbo.

\section{AGRADECIMENTOS}

Os autores agradecem aos órgãos de fomento à pesquisa CAPES e CNPq pelas bolsas fornecidas para A. Vanz e N. Mirlean.

\section{REFERÊNCIAS}

1. World Health Organization; Guidelines for Air Quality, WHO: Geneva, 1999.

2. Cochran, J. K.; Frignami, M.; Salamanca, M.; Bellucci, L .G.; Guerzoni, S.; Mar. Chem. 1998, 62, 15.

3. Stern, A. C.; Air Pollution, Academic Press: London, 1977.

4. Fundação Estadual de Proteção Ambiental Henrique Roessler-RS; Avaliação de Dados da Qualidade do Ar Médios em Rio Grande/RS, FEPAM: Porto Alegre, 1995, p. 42.
5. U.S. Environmental Protection Agency; Quality Assurance Handbook for Air Pollution Measurements Systems: Ambient Air Specific Methods, USEPA: Washington, DC, 1987, vol. I and II.

6. Mirlean, N.; Moraru, K.; Nastas, G.; Ecologo-geochemical Atas of City Kishinev, Stiintsa: Kishinev, 1992.

7. Saet, Y.; Environmental Geochemistry, Nedra: Moscow, 1990.

8. Vanz, A.; Mirlean, N.; Baisch, P.; Anais do VII Congresso Brasileiro de Geoquímica e V Congresso de Geoquímica dos Países de Língua Portuguesa, Porto Seguro, Brasil, 1999.

9. Perelman, A. I.; Geochemistry of Epigenesis, $2^{\text {nd }}$ ed., Premium Press: New York, 1989.

10. Mirlean, N.; Matouzenko, G.; Banar, S.; Izv. Akad. Nauk MSSR 1991, 2 , 105.

11. Ministry of Health of the USSR; Guidelines for Urban Environment Quality, MH: Moscow,1988.

12. Brigoni, S. F.; Anais do XII Congresso Brasileiro de Engenharia Sanitária e Ambiental, Camboriú, Brasil, 1983.

13. Baisch, P.; Mirlean, N.; Vanz, A.; Anais da XI Semana Nacional de Oceanografia, Rio Grande, Brasil, 1998.

14. Loring, D. H.; Intercalibration for Metals in Marine Sediments, Report Dep. Fish. \& Oceans: Dartmouth, 1986.

15. Tagliani, C. R. A.; Dissertação de Mestrado, Universidade do Vale do Rio dos Sinos, Brasil, 1997.

16. Cantanho, A. D. A.; Artaxo, P.; Atmos. Environ. 2001, 29, 4889.

17. Echalar, F.; Artaxo, P.; Martins, J.V.; Yamasoe, M.; Gerab, F.; Maenhaut, W.; Holben, B.; Journal of Geophysical Research-Atmospheres 1998, 103, 849.

18. Silva, E. V.; Paiva, R. P.; Wasserman, J. C.; Lacerda, L. D.; J. Braz. Chem. Soc. 1998, 9, 482. 